\title{
Design and implementation of remote image processing system for space launch site
}

\author{
Ma Li, Wang Bai Yan, Liu Tao, WangYu Chao, Xiang Yu, Li Peng \\ (Xichang Satelite Launch Center, Sichuan Xichang 615000, China )
}

\begin{abstract}
Telemetry image has the characteristics of intuitive image in the process of rocket flight. Through real-time acquisition of rocket flight video image, it can provide the working status of key nodes in the process of rocket flight, and provide intuitive decision-marking auxiliary information for commanders. This paper analyzes the design content of the image processing system of the space launch site from the aspects of image transmission mechanism, information flow, image data processing and image decoding, so as to provide technical basis for the image receiving, transmission and decoding process in the engineering practice of the image processing system.
\end{abstract}

\section{Introduction}

Image measurement technology has been widely used in aerospace domestically and abroad. Real time transmission of various image information between spacecraft and ground station can be seen on various missiles, reconnaissance satellites, space shuttles and launch vehicles. Currently, multiple cameras are installed in each cabin and key parts of the rocket to synchronously collect the image data on the rocket in the space launch site ${ }^{[1]}$. Some crucial actions such as fairing separation, booster separation, interstage separation and satellite rocket separation can be observed by image measurement technology, and the working conditions of each cabin can be viewed in real time.

After being compressed, the telemetry image data can form a telemetry data frame with other measurement parameters on the rocket. Then it can be sent to the ground through microwave signal. The telemetry data frame received by the ground telemetry equipment is sent to the image processing system of arrow telemetry in the way of multicast, and the telemetry image data can be converted into video image through path selection and decoding for the decision-making and technical personnel to watch. The information flow of arrow remote image processing system is shown in the figure below.
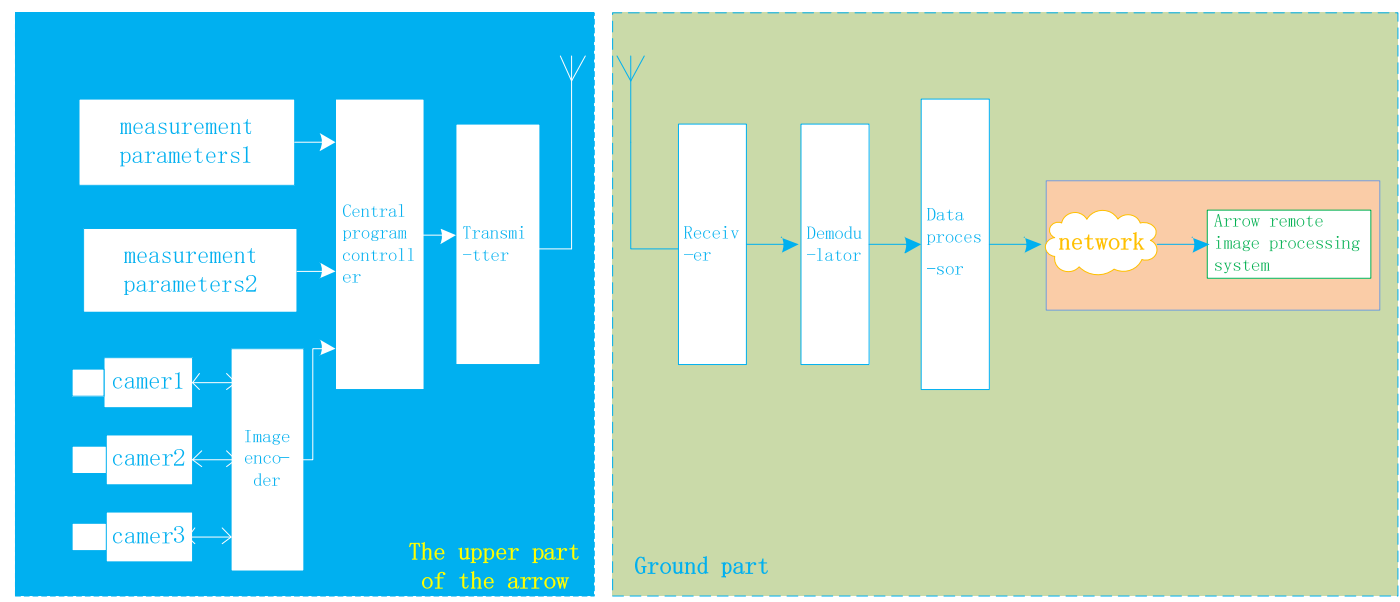

Fig.1 Information flow chart of remote arrow image processing system

\section{Composition of Remote Image Processing System}

The arrow remote image processing system adopts dual redundancy hot standby mode, and its information flow is shown in Figure 2. The system uses multicast mode ${ }^{[2]}$ to receive the telemetry full frame source code and path selection data in real time, processes the image data, verifies the correctness of the image data, and stores it into 
the temporary image buffer. Through the embedded system $^{[3]}$, it completes the image data packet, real-time decompression, image amplification, image filtering, video coding, output ${ }^{[4]}$, and realizes the real-time display of arrow remote image.

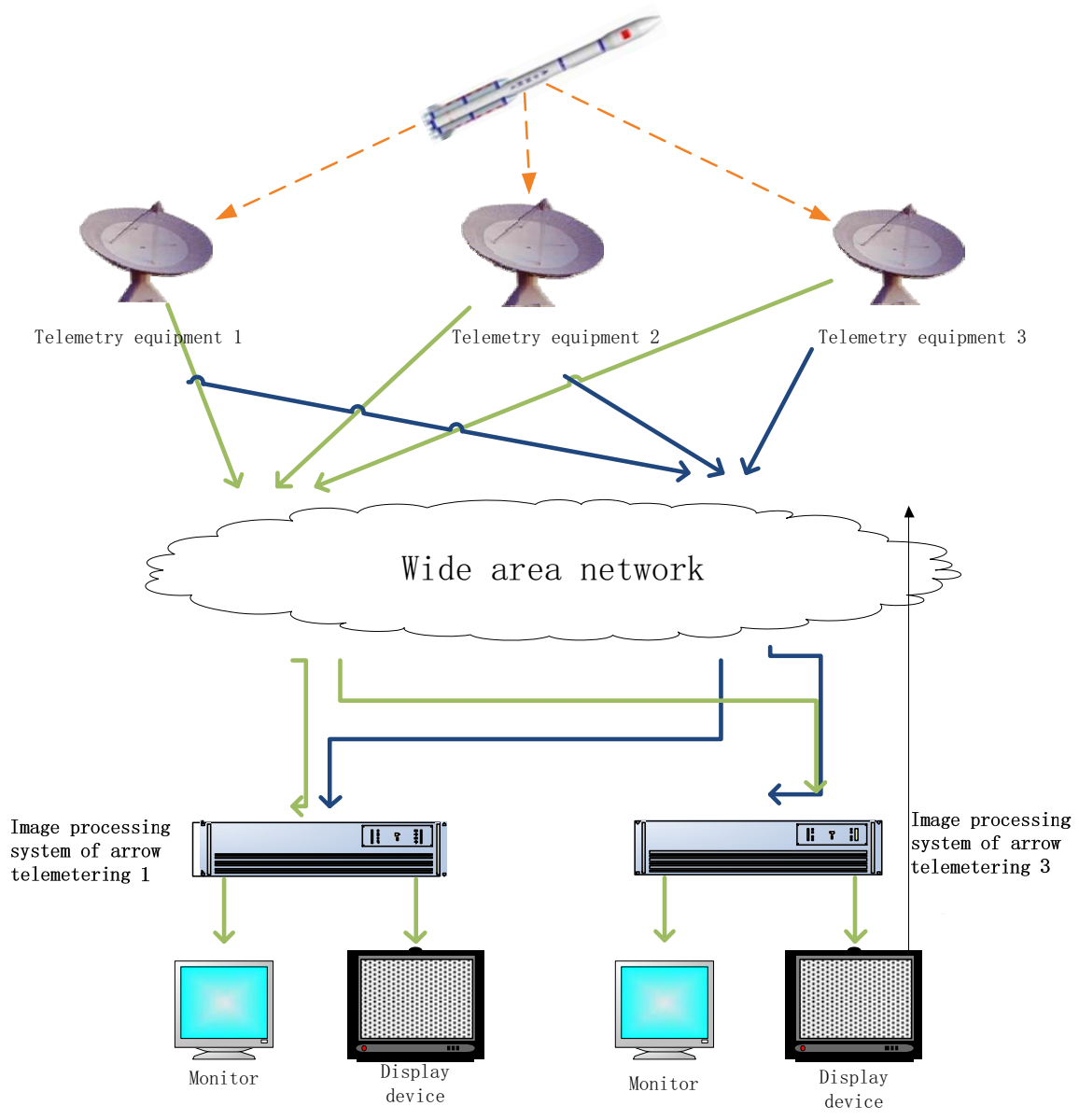

Fig.2 Hardware composition of telemetry image decoding system

\section{Design of Image Processing System for Remote Arrow}

The archery remote image processing system with multiple telemetry image data streams mainly includes four functions: real-time source code receiving, image data processing, image-data sending and decoding. The system uses multicast to receive data sent by telemetry equipment in real time. The equipment information flow of arrow remote image processing system is shown in Figure 3.

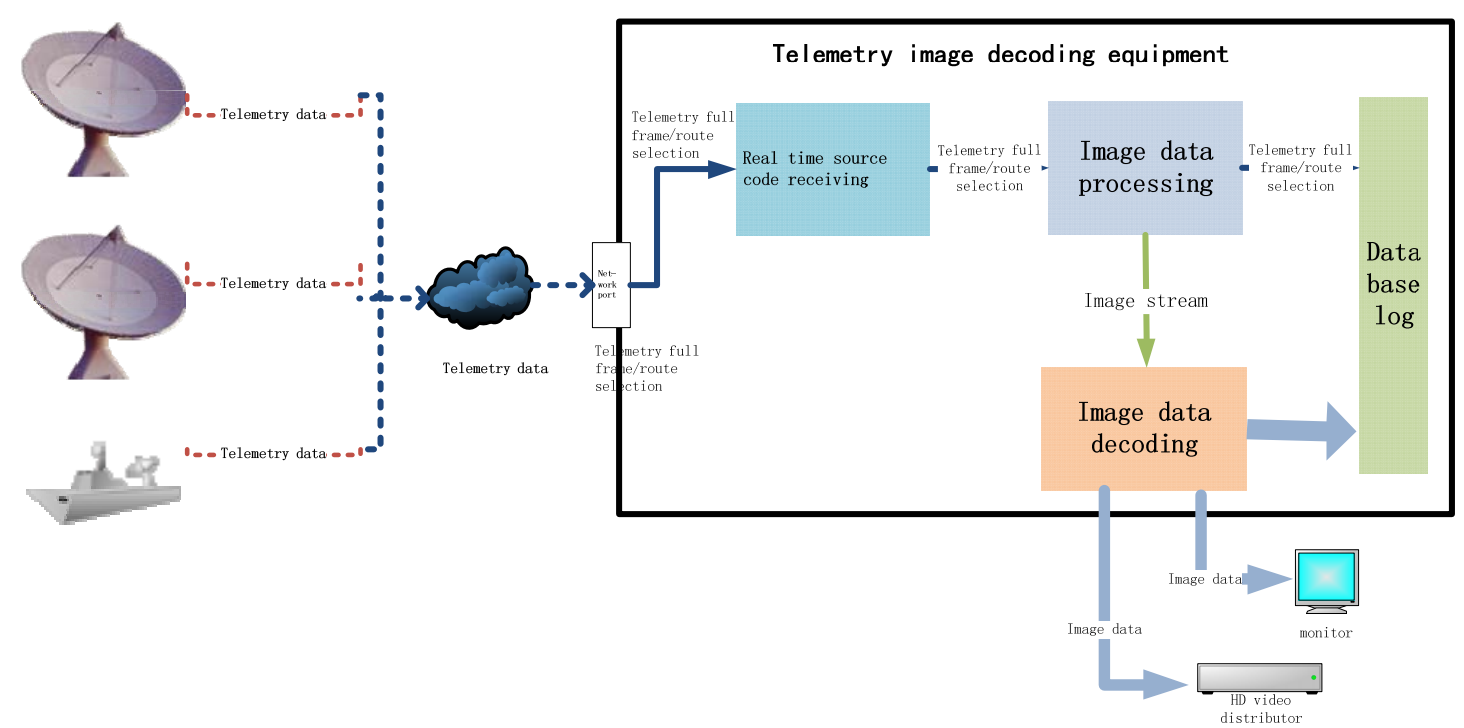

Fig.3 Information flow chart of telemetry image decoding equipment 


\subsection{Real Time Receiving of Source Code}

The source code real-time receiving module first receives the telemetry source data in real-time from A and B planes of multiple telemetry devices in the form of multicast. The data automatic splicing algorithm is used to realize the asynchronous splicing between the site and the plane to ensure the correctness and reliability of the data flow. Using the precise timer technology ${ }^{[5]}$, according to the navigation area time planning in the script, the receiving station can be automatically switched. The block diagram of the design of the source code real-time receiving module is shown in Figure 4.

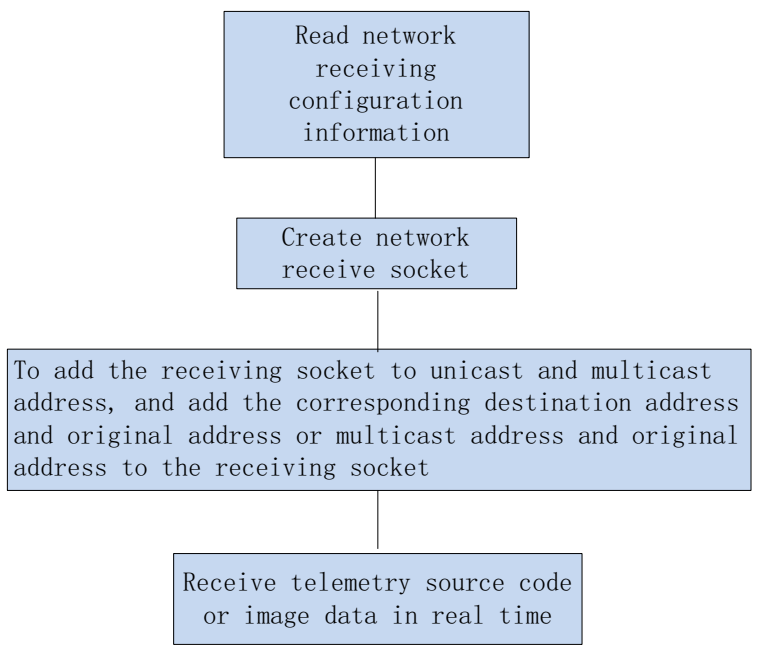

Fig.4 design block diagram of data receiving module

The flow chart of image data optimization is shown in Figure 5. Each route taking host can receive the first and second plane data streams at the same time. After receiving the two channels of data, the two channels of data are judged respectively, and the two channels of data sources are selected based on frame continuity. If both channels of data are continuous, the first channel is preferred by default. If the two channels are not continuous, the first data is used by default.

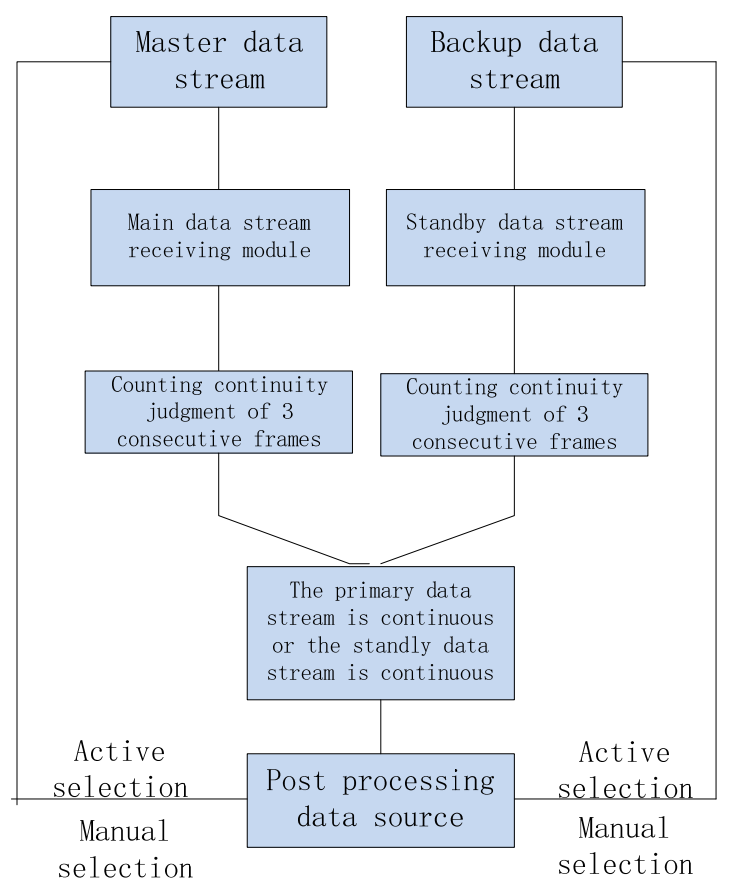

Fig.5 image data optimization process

\subsection{Image Data Processing}

The image data processing module selects the path of the telemetry source code received by the source code realtime receiving module ${ }^{[6]}$, and provides the processed data to the image data transmission module. Its specific functions include: 


\section{(1) Verification Function of Data Source}

Verify the correctness of the data format to ensure the reliability of the received data. The software can automatically identify the data transmission protocol and judge that the data is telemetry source data or image data [7]. According to the result of discrimination, the corresponding processing program is run for different data sources.

According to the data transmission protocol, within the specified time, the route taking host receives the telemetry frame signal in real time. For the same frame data, the length of the data field between the image data and the telemetry source code is different. After the software analyzes the received data, the length of the data field can be automatically identified to identify the telemetry full frame data or the telemetry path picking data.

(2) Data Route Selection Function Design

According to the telemetry data channel bound before the task, the telemetry route selection software selects the optimized source data and stores it into the temporary image buffer.

\section{(3) Data Storage Function}

The remote sensing software stores the image data and source data before the road selection. The image data is stored in a buffer for subsequent adaptive transmission.

\subsection{Image Data Transmission}

The image data sending module uses UDP protocol to send image data to decoder by unicast. Due to the instability of the network and other reasons, the image processing system may receive a large number of telemetry source data instantaneously. The telemetry route selection software has the function of precise timing. The adaptive adjustment technology can make the image data sent to the image decoding equipment smoothly and evenly. The design of image data transmission software is shown in Figure 6.

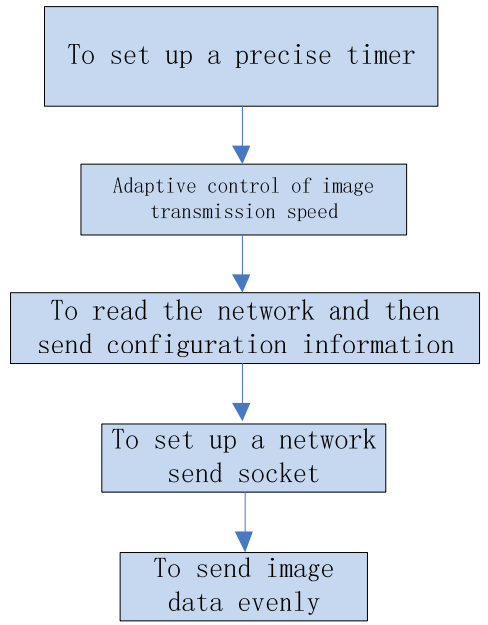

Fig.6 design of image data transmission software

\subsection{Image Data Decoding}

The image data decoding module receives the compressed image data in real time and sends it to the monitor for display after error correction, decoding and amplification. It mainly includes five modules: compressed image data reading module, channel decoding module, preprocessing module, image decompression module, display and playback module. The functional structure of image decoding software is shown in Figure 7. Its specific functions include: (1) receiving compressed image data in real time according to configuration information ${ }^{[8] ~[9] ; ~(2) ~}$ using video coding technology ${ }^{[10]}$ to correct the compressed image data; (3) decoding the compressed image data to restore the real image data by using a special decoder; (4) forming a data format meeting the standard requirements after post-processing the image data Go to the monitor for display.

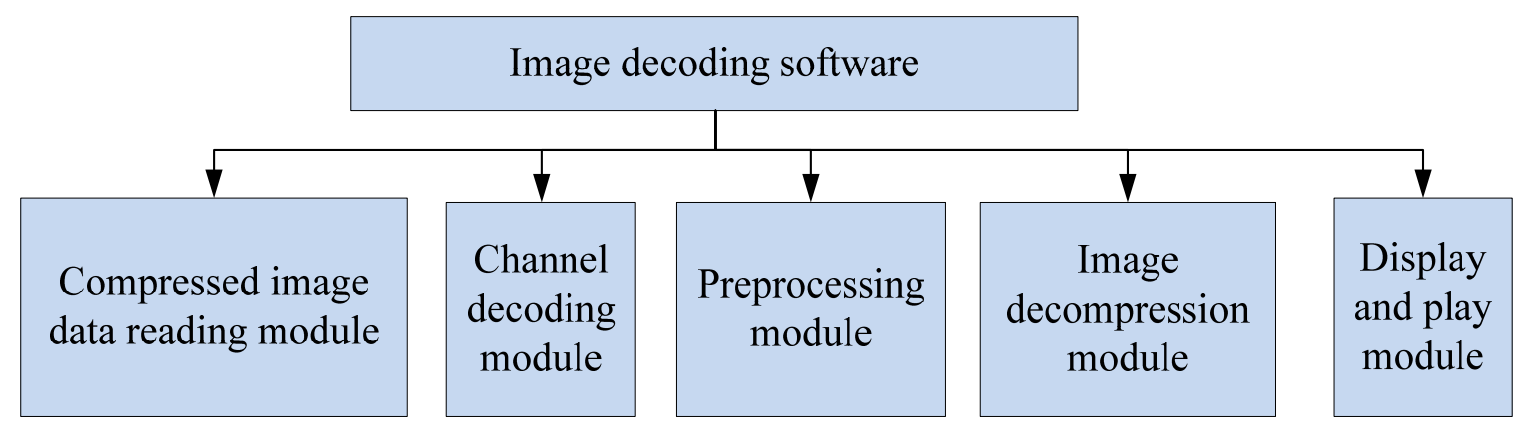

Fig.7 Functional structure of image decoding software

\section{Experimental Verification}

The rocket remote image can provide real-time and accurate rocket firing, booster separation, primary and secondary separation, fairing separation, secondary and tertiary separation and satellite rocket separation. Figures
8-1 and 8-2 show the real-time telemetry images of a certain mission, from which the arrow remote images of some key nodes are intercepted. Suppose the rocket is abnormal in the flight process, it can provide the commander with intuitive decision-making auxiliary information by comparing the remote arrow images of the same type of mission. 


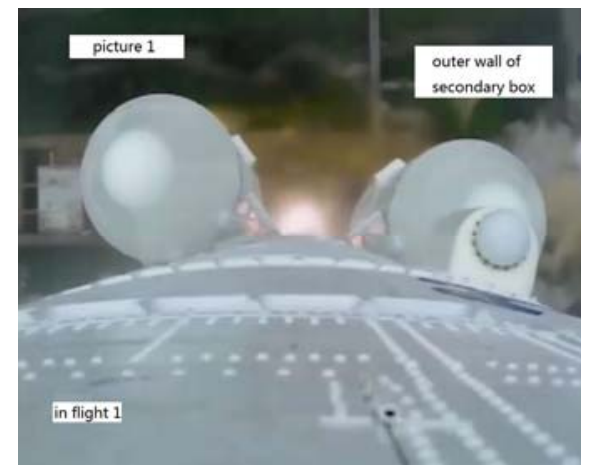

Fig.8-1 Real-time telemetry images

\section{Conclusion}

The archer remote image processing system mainly receives the telemetry full frame source code and path selection data in real time by multicast, processes the telemetry image data, verifies the correctness of the image data, and stores it into the temporary image buffer. The high-precision timer is used to uniformly send to the realtime image decoder to realize the function of decoding the telemetry full frame or path picking data into the telemetry image. The system can provide the working status of key nodes in the process of real-time rocket flight, and provide intuitive decision-making auxiliary information for commanders. At present, the system is working well with all functions realized, including the stable and reliable image decoding, which can meet all work requirements.

\section{Reference}

1. Li Ya,Automatic Splicing \&Optimization Method on Multiple Telemetry Image Data Streams [J] Electronic Technology \& Software Engineering,2018, 09: 153

2. Xiao Ming, Rong Wei Jun, Li Jing Jing. Design of telemetry image forwarding software based on $\mathrm{C}++$ Builder 6.0 and multicast technology[J], 2019,02:143-147

3. Li Sheng Lan,Jiang Hong Xu,Fu Wei Jian.Design of DMA controller for multi-channel transmission

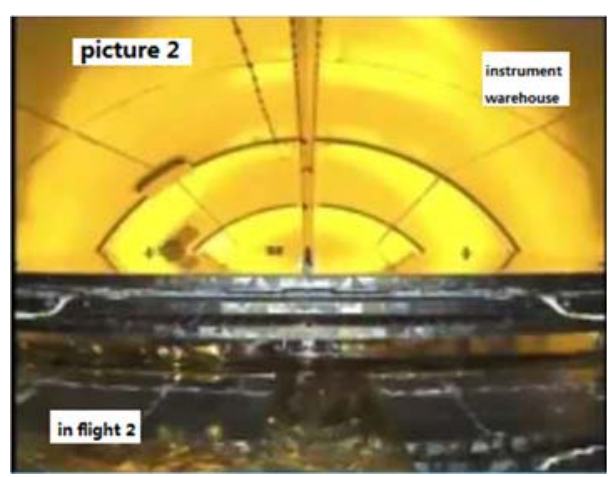

Fig.8-2 Real-time telemetry images

system based on PCIe[J]. Journal of Computer Applications, 2017, 37(03): 691-694.

4. Design and Implementation of A Model Load Image JPEG2000 Decompression System. Yanshan University of Electronic Science and technology, 2019: 8-19

5. Li Bo,Li Ya'nan,Li Jian.Realization Video-imagedata Acquisition and Accomplish Based on FPGA+DSP Framework Asynchronous FIFO.[J]. Ordnance Industry Automation, 2016, 35(09):31$34+40$.

6. SHI Zhong。FPGA- based DVI Data Storage and Image Processing System [J】, Journal of Changchun University, 2016,26(08):1-8.

7. Lin Kun. The FPGA Design\&Implementation of High-speed DAC Based on the PCIE Bus[D].Cheng $\mathrm{Du}$ :Master's thesis of University of Electronic Science and technology, 2013: 34-44.

8. MU Ke,LI Wen-na. The Medical Image Compression Method Based on Fuzzy C-mean Clustering[J]. Control Engineering of China, 2016, 23(05): 706-710

9. Zhao Yili,Xu Dan,Qian Wenhua,Zhang Yan,Fast Image Blending Using Run-length Encoding and SIMD Instruction Set[J],Journal of Computer-Aided Design \& Computer Graphics,2016,28(04):623-631.

10. Xiang Bing,Gao Yu.Application of Interweavingcoding in Image Telemetry[J],Modern Electronics Technique,2006,23:36-43. 